\title{
亚
}

\section{Volumetric Capnometry, more than end-tidal carbon dioxide}

Mia Shokry ${ }^{1}$, Kimiyo Yamasaki ${ }^{2}$

DOI: https://doi.org/10.53097/JMV.10032

Cite: Shokry M, Yamasaki K.Volumetric capnometry, more than end-tidal carbon dioxide. J Mech Vent 2021; 2(3):112-113.

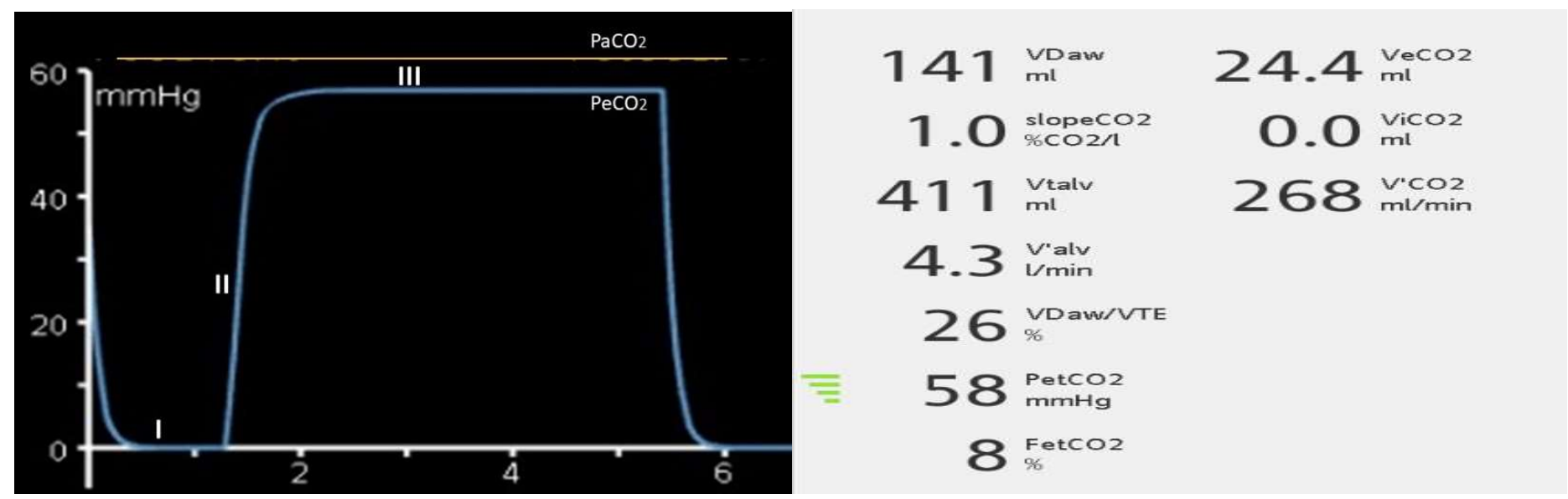

Volumetric capnography: $\mathrm{x}$-axis is time in seconds, y-axis: exhaled $\mathrm{CO}_{2}$ in mmhg. I: Phase 1, II: phase 2, III: phase 3. PaCO 2 : partial pressure of carbon dioxide in blood, $\mathrm{PeCO} 2$ : partial pressure of $\mathrm{CO}_{2}$ in exhaled volume.

$\mathrm{FeCO}_{2}$ : fractional concentration of carbon dioxide, Valv: alveolar minute ventilation/minute, VDaw: dead space in ml, VDaw/VTE: ratio of dead space to alveolar tidal volume, $\mathrm{VeCO}_{2}$ : carbon dioxide production per minute, Vtalv: alveolar tidal volume in $\mathrm{ml}$.

Authors

1. Mia Shokry, premed student, University of Southern California, California, USA

2. Yamasaki K. RRT, Adventist Health Castle Medical Center, Hawaii, USA

Corresponding author: miashokry@gmail.com

Conflict of interest/Disclosures: None

Funding: None

Journal of Mechanical Ventilation 2021 Volume 2, Issue 3

This open-access article is distributed under the terms of the Creative Commons Attribution Non-Commercial License (CC BY-NC) (http://creativecommons. $\mathrm{org} /$ licenses/by-nc/4.0/), which permits reuse, distribution and reproduction of the article, provided that the original work is properly cited and the reuse is restricted to 
Monitoring the exhaled caron dioxide pressure, known as endtidal $\mathrm{CO} 2\left(\mathrm{ETCO}_{2}\right)$ has become the standard of care during anesthesia, intensive care units, and during cardiac arrest resuscitation.

However, volumetric capnometry provides much more useful information other than the $\mathrm{ETCO}_{2 .}{ }^{1,2}$ The parameters provided are:

\section{ETCO2 (PetCO $)$ :}

Is the partial pressure of carbon dioxide at the end of exhalation in mmhg. It gives an indirect estimate of arterial carbon dioxide partial pressure $\left(\mathrm{PaCO}_{2}\right)$. In normal lungs, the PetCO2 is 2-5 mmhg below $\mathrm{PaCO}_{2}$.

However the difference could be much higher in diseases with high ventilation-perfusion mismatch $(\mathrm{V} / \mathrm{Q})$ and dead space.

\section{Alveolar tidal volume (Vtalv):}

Is the effective gas in the alveoli involved in ventilation measured in $\mathrm{mL}$. The value is obtained from the full tidal volume - the Dead space.

This value is important especially in setting PEEP to maximize the Vtalv (decreasing the value during decremental PEEP could point to more alveoli collapsing during the decrease in PEEP). Additionally, this value is important during the correction of hypercapnia as increasing the respiratory rate and tidal volume might increase the dead space without increasing the alveolar tidal volume.

\section{Alveolar minute ventilation (Valv):}

Is the alveolar tidal volume $\mathrm{X}$ respiratory rate

\section{Dead space (VDaw):}

Is the portion of the tidal volume that does not participate in gas exchange in $\mathrm{mL}$. It is divided into:

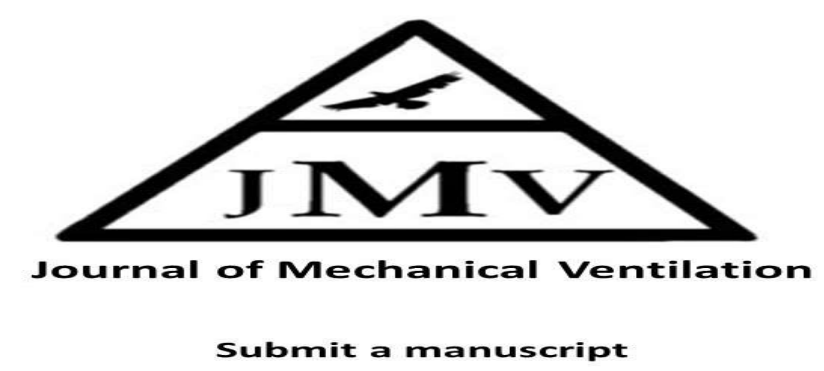

https://Www.journalmechanicalventilation -com/submit-a-manuscript/
- Anatomic dead space: conduction portion of the respiratory system.

- Functional dead space: alveolar dead space comprises alveoli where gas exchange does not occur (i.e., non-perfused alveoli).

- Physiologic dead space includes the anatomical space and functional dead space

The value is calculated from the graph. This value is important in setting PEEP and might aid in diagnosing other diseases that can have high dead space like obstructive airway diseases and even ARDS.

\section{Dead space fraction (VDaw/VTE):}

Is the percent fraction of the dead space to the exhaled tidal volume. Normally $25-30 \%$. Similarly it can be helpful in setting PEEP and diagnosis of diseases of high dead space.

\section{Carbon dioxide production $\left(\mathrm{VCO}_{2}\right)$ :}

Is the amount of carbon dioxide exhaled from the body per unit time $\mathrm{ml} / \mathrm{min}$, normally around $200 \mathrm{ml} / \mathrm{min}$. This value is usually obtained during pulmonary function tests but can be useful during mechanical ventilation for adjusting PEEP, diagnosing the etiology of hypercapnia like hypermetabolic states or overfeeding, and can be used to estimate the energy expenditure and calculate nutritional support required.

\section{References}

1. Kreit JW. Volume capnography in the intensive care unit: physiological principles, measurements, and calculations. Ann Am Thorac Soc 2019; 16(3):291-300.

2. Volumetric capnography. E-book, Hamilton medical @ https://www.hamiltonmedical.com/en_US/Solutions/Volumetric-capnography.html. Accessed August 2021.

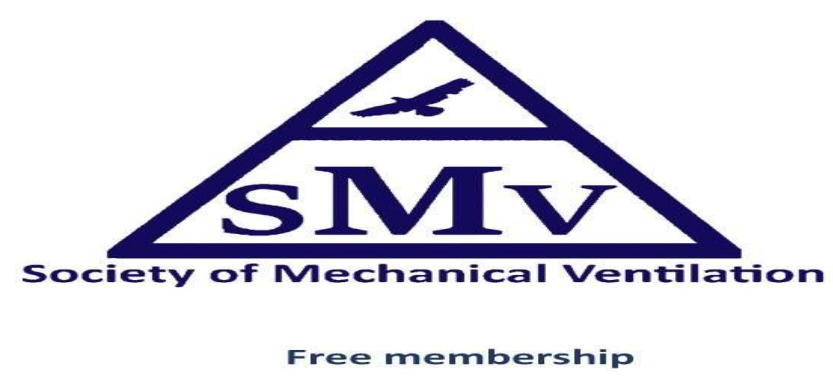

https://societymechanicalventilation.org /membership/ 